\title{
Julian, Kaiser und Philosoph
}

\author{
Klaus Bringmann
}

In der Zeit der kurzen, nur 20 Monate von November 361 bis Juni 363 n.Chr. dauernden Alleinherrschaft Julians ehrte Aelius Claudius Dulcitius, der Prokonsul der Provinz Asia, ${ }^{1}$ den Kaiser mit der Errichtung von Statuen und Meilensteinen. Die Basis einer der in Ephesos aufgestellten Statuen trug folgende Inschrift:

$\mathrm{D}$ (omino) $\mathrm{n}$ (ostro) $\mathrm{Fl}$ (avio) $\mathrm{Cl}$ (audio) Iuliano, virtutum omnium magistro,

philosophiae principi,

venerando et

5 piissimo imperatori,

victoriosissimo Augusto,

omnium barbararum

gentium debellatori

Ael(ius) Cl(audius) Dulcitius

$10 \mathrm{v}$ (ir) c(larissimus), procons(ul) Asiae, vic(e) s(acra) $\operatorname{cog}$ (noscens),

$d$ (evotus) n(umini) maiestatiq(ue) eius. ${ }^{2}$

Die deutsche Übersetzung lautet:

Unserem Herrn Flavius Claudius Iulianus, dem Lehrer [oder: Meister] aller Tugenden, dem Prinzeps der Philosophie,

dem verehrungswürdigen und

5 frömmsten Imperator, dem siegreichsten Augustus, dem Überwinder aller barbarischen Völkerschaften [widmet diese Statue]

Aelius Claudius Dulcitius,

10 Exzellenz, Prokonsul von Asia,

$1 \mathrm{Zu}$ seiner Person und Karriere siehe Jones, Arnold Hugh Martin/Martindale, John R./Morris, John, Prosopography of the Later Roman Empire I, A.D. 260-395, Cambridge 1971, 274, sub voce Aelius Claudius Dulcitius 5 (die Prosopographie wird unten mit der Abkürzung Prosopographie I zitiert). Die Statthalterschaft der Provinz Asia, die Dulcitius in der Zeit der Alleinherrschaft Julians innehatte, scheint Höhepunkt und Ende seiner Karriere gewesen zu sein.

2 Conti, Stefano, Die Inschriften Kaiser Julians (Altertumswissenschaftliches Kolloquium 10), Stuttgart 2004, Nr. 26. 
Richter in Appellationsverfahren,

in Ergebenheit gegenüber seiner göttlichen

Wirkkraft und Majestät.

In Pergamon ließ derselbe Aelius Claudius Dulcitius an einer von ihm gestifteten Basis eine Inschrift mit folgendem Wortlaut anbringen:

$\mathrm{D}$ (omino) n(ostro) Fl(avio) C[1(audio)] [[Iulian]]o,

domino [to]tius orbis,

filosofi[ae] magistro,

venera[nd]o principi,

5 piissimo [imp]eratori,

victorios[iss]imo Augusto,

propagatori libertatis

et rei publ[i]cae

Ae(lius) Cl(audius) Dulc[i]tius,

$10 \mathrm{v}$ (ir) c(larissimus), procon[s(ul)], vic(e) s(sacra) aud(iens), d(evotus) n(umini) m(aiestati)q(ue) su[ae?]. ${ }^{3}$

Die Übersetzung lautet:

Unserem Herrn Flavius Claudius Iulianus, dem Herrn des gesamten Erdkreises, dem Lehrer [oder: Meister] der Philosophie, dem verehrungswürdigen Prinzeps,

5 dem frömmsten Imperator, dem siegreichsten Augustus, dem Beförderer von Freiheit und Gemeinwohl [stiftet dies]

Aelius Claudius Dulcitius,

10 Exzellenz, Prokonsul von Asia,

Richter in Appellationsverfahren,

in Ergebenheit gegenüber seiner göttlichen

Wirkkraft und Majestät.

Beide Inschriften durchbrechen die Konvention der einem römischen Kaiser der Spätantike beigelegten Titulatur und öffnen den Blick auf das Besondere und Außerordentliche, das Julians Kaisertum in seinem eigenen Selbstverständnis und in der Wahrnehmung seiner Anhänger auszeichnete. Gewiss: Die üblichen Titel eines römischen Kaisers wie Prinzeps, Imperator, Augustus begegnen auch hier, und an der Stelle individueller Siegerbeinamen erscheinen pauschale Formeln, omnium barbararum gentium debellatori oder victoriosissimo Augusto, aber völlig aus dem Gewohnten fallen die Prädikationen omnium virtutum magistro, philosophiae principi oder filosofiae magistro. Sie sind Ehrentitel eines Philosophen und entsprechen der Würdigung, die ein gewisser Iulius Iulianus (nicht zu verwechseln mit dem Onkel Kaiser Julians) zu Beginn des vierten Jahrhunderts

3 Conti (Anm. 2), Nr. 28. 
n.Chr. auf seinem Grabstein an der Via Latina in der Nähe Roms erhalten hat: viro magno, philosopo [sic] primo. ${ }^{4}$ Was Kaiser Julian anbelangt, so scheint mir die Verbindung princeps philosophiae besondere Aufmerksamkeit zu verdienen. Zunächst liegt die Annahme nahe, dass Julian damit die höchste Autorität in der Philosophie zugesprochen wird, etwa in dem Sinne, wie Cicero in De natura deorum Sokrates einmal den princeps philosophiae nennt. ${ }^{5}$ Nun bezeichnet das Wort princeps aber auch den römischen Kaiser. In diesem Sinn ist es in der Inschrift Nr. 28 Conti gebraucht. ${ }^{6}$ Möglicherweise ist daran zu denken, dass in Nr. 26 der Verbindung principi philosophiae eine Bedeutungsambivalenz zugrunde liegt und Julian zugleich als höchste Autorität in der Philosophie und als Kaiser aus ihrem Geist bezeichnet wird. Wie immer man aber über die Möglichkeit einer bewusst gewählten Bedeutungsambivalenz denken mag: Aus Iasos, einer Stadt der Asia benachbarten Provinz Caria, besitzen wir eine griechische Inschrift, die

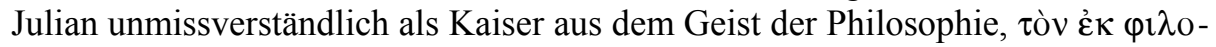
$\sigma o \varphi i ́ \alpha \varsigma \beta \alpha \sigma \imath \lambda \varepsilon v ́ o v \tau \alpha$ apostrophiert. Der griechische Text hat folgenden Wortlaut:

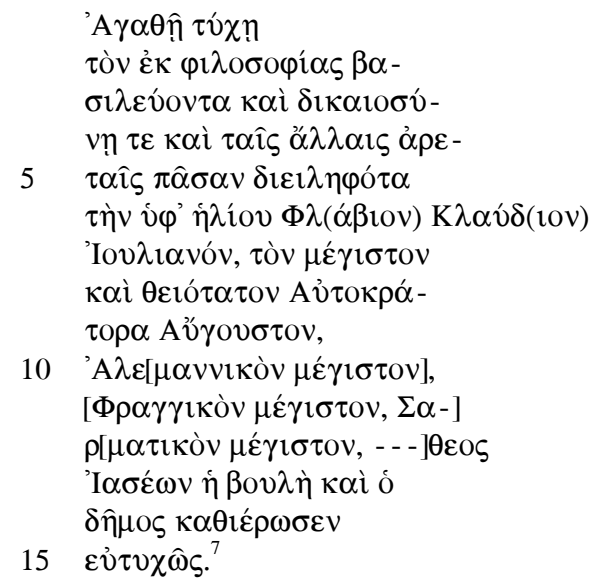

Der Text, mit dem Rat und Volk der griechischen Stadt eine Statue des Kaisers weihten, ist höchstwahrscheinlich von einem Neuplatoniker, einem Anhänger und

4 Corpus Inscriptionum Latinarum VI, Nr. 9785 = Inscriptiones Latinae Selectae II 2, Hg. Hermann Dessau, Nr. 7778.

5 Cicero, De natura deorum II, 167: ... siquidem satis a nostris [sc. Stoicis] et a principe philosophiae Socrate dictum est de ubertatibus virtutis et copiis.

6 Vgl. auch die stadtrömische Marmortafel mit der Widmung an Julian in: Conti (Anm. 2), Nr. 115: [--- p]rincipi /[d(omino nostro Iulia]no victori / (ac trium]f(atori) semp(er) Aug(usto).

7 Conti, Stefano (Anm. 2), Nr. 34. Die bei Conti abgedruckte Ergänzung der Zeilen 10-12 ist schwerlich richtig. In den fast vollständig eradierten Zeilen könnte der Name eines Magistrats gestanden haben, der aus welchem Grund auch immer getilgt worden ist. Dafür spräche auch die stehengebliebene Endsilbe -theos, ein häufiges Namensende im Griechischen: vgl. Contis Kommentar dortselbst 82 mit Literatur. 
Gesinnungsgenossen Julians, verfasst worden und folgt im ersten Teil, in den Prädikationen der Zeilen 2-5, unverkennbar dem neuplatonischen Konzept, dem Julian sein Kaisertum unterstellte. Die Übersetzung lautet:

Mit gutem Glück.

Den Kaiser aus (dem Geist) der Philosophie, der mittels Gerechtigkeit und allen übrigen

Tugenden die in ihrer Gesamtheit unter (der

$5 \quad$ Herrschaft) des Sonnengottes stehende (Tugend) in ihren Teilen erfasst, Flavius Claudius

Iulianus, den größten

und göttlichsten Imperator

Augustus,

10 [den größten Alamannensieger, größten Frankensieger, größten

Sarmatensieger]---theos (?)

haben Rat und Volk von

Iasos geweiht

$15 \mathrm{zu}$ gutem Gelingen.

Wie es scheint, ist mit dieser verkürzten Aussage auf die Rolle des Sonnengottes als Ursprung und Mittler des Guten angespielt, der den Seelen der Menschen Anteil an der geistigen Kraft des Erkennens und eine partielle Teilhabe an der Idee des Guten gibt. ${ }^{8}$ Die Philosophie aber ist das Medium, das ihn in die Lage versetzt, sich der göttlichen Vermittlung zu öffnen und die einzelnen Tugenden in der sublunaren Welt zu bewähren. Julian hatte sich der neuplatonischen Philosophie verschrieben, den Sonnengott, dem er im Winter 362/63 n.Chr. zu seinem Fest eine von Iamblichos' Abhandlung über die Götter inspirierte Lobrede widmete, wählte er zu seinem Schutzgott, und er erhob, wie noch näher zu zeigen sein wird, den Anspruch, Kaiser aus dem Geist der wahren, das heißt: der neuplatonischen Philosophie zu sein. Der Verfasser des Inschriftentexts war mit dem Programm, unter das Julian seine Alleinherrschaft gestellt hatte, offenbar wohlvertraut, und die Vermutung liegt nahe, dass er zumindest in den weiteren Umkreis des neuplatonischen Philosophenzirkels gehörte, dem Julian sich selbst zurechnete.

Es ist sicher kein Zufall, dass die drei zitierten Inschriften in dem Teil des Römischen Reiches gefunden worden sind, wo Julian in der ersten Hälfte der fünfziger Jahre des vierten Jahrhunderts Schulen der Neuplatoniker besuchte und dem Christentum, in dem er erzogen worden war, abschwor. ${ }^{9}$ Er begann sein

8 Vgl. Iulianus, Oratio IV, 151 C-D über König Helios als Urheber der geistigen und moralischen Seelenkräfte; vgl. 145 C.

9 Er selbst hat gegen Ende des Jahres 362 in einem Brief an die Alexandriner seine Bekehrung in das Jahr 351 gesetzt: Iulianus, Epistula 111, 434 D-435 C (nach der Zählung in: Imp. Caesaris Flavii Claudii Iuliani Epistulae Leges Poemata Fragmenta Varia, Hg. Joseph Bidez/Franz Cumont, Paris 1922; im Folgenden zitiert mit der Abkürzung Bidez/Cumont). Dieses eindeutige Selbstzeugnis spricht gegen den Versuch, Julian eine langdauernde religiöse Entwicklung zuzu- 
Studium in Pergamon in der Schule des Iamblichschülers Aidesios, aber er wechselte mit fliegenden Fahnen nach Ephesos, wie Eunapios versichert, ${ }^{10}$ als er erfuhr, dass dort Maximus, ein Schüler des Aidesios, wirkte, der nicht nur als groBer Lehrmeister in philosophischer Theorie galt, sondern vor allem wegen seiner übernatürlichen Fähigkeiten in der Theurgie, der praktischen Kunst, mit den Göttern zu kommunizieren, berühmt war. ${ }^{11}$ Was für Julian die Studentenzeit in Kleinasien bedeutet hat, ist später in einem Nachruf von Libanios, auch er ein praktizierender Verehrer der Götter aus neuplatonischem Geist und Bewunderer des Kaisers, so beschrieben worden:

Julian wurde durch die Begegnung mit Menschen gerettet, die von Platons Philosophie erfüllt waren. Durch sie hörte er von den Göttern und Dämonen, die in Wahrheit das Universum geschaffen haben und es erhalten. Von ihnen lernte er, das Wesen der Seele, ihre Herkunft und ihre Zukunft zu erkennen, das Gesetz ihres Sturzes und ihres Aufstiegs, was sie herabzieht und was sie aufsteigen lässt, was Gefangenschaft und was Befreiung für sie bedeutet, wie sie jene umgehen und diese erreichen kann. Danach tat er alle Torheiten von sich ab, an die er bis dahin geglaubt hatte, um dafür dem Licht der Wahrheit Raum zu geben - so, wie man in einem Tempel die vorher mit Schmutz besudelten Götterbilder neu aufstellt. ${ }^{12}$

Julian hat glaubhaft versichert, dass er es vorgezogen hätte, als Privatmann im Verborgenen seinen Studien zu leben, ${ }^{13}$ aber seine Zugehörigkeit zum Kaiserhaus ließ das nicht zu. Im November 355 n.Chr. erhob sein Vetter, Kaiser Constantius

schreiben, die erst mit der Gewinnung der Alleinherrschaft im November 361 ihren Endpunkt, die Bekehrung zum Heidentum, gefunden hätte: so jedoch Rosen, Klaus, Kaiser Julian auf dem Weg vom Christentum zum Heidentum, in: Jahrbuch für Antike und Christentum 40 (1997), S. 126-146. Freilich verbarg Julian seinen Abfall vom Christentum, solange Constantius lebte. Noch im Januar 361 besuchte er aus taktischer Rücksicht zum Epiphaniasfest in Vienna die Kirche: „Alle wollte er für sich gewinnen, ohne dass jemand sich entgegenstellte, und so gab er vor, Anhänger der christlichen Religion zu sein, obwohl er schon früher insgeheim abtrünnig geworden war. Nur wenige waren an den heimlichen Handlungen beteiligt, wenn er sich der Opferund Vogelschau sowie allen sonstigen Zeremonien hingab, welche die Verehrer der Götter stets vollzogen haben. Um das zu verheimlichen, ging er an dem Fest, das die Christen im Januar begehen und Epiphanias nennen, in die Kirche. Hier betete er die Gottheit öffentlich an und entfernte sich dann"(Ammianus Marcellinus, Res gestae XXI,2,4 f.).

10 Eunapius, Vitae sophistarum VII, 2,1-13 (474 f.). Aidesios überwies Julian mit Rücksicht auf sein hohes Alter an seine Schüler Chrysanthios und Eusebios von Mynda. Dieser war ein Gegner theurgischer Praktiken und pflegte seine Vorlesungen mit der Bemerkung zu beenden, ,dass die magischen Handlungen nur Täuschung der Sinne und Blendwerk seien, Zaubertricks von Wundertätern und anderen Verführern und Wahnsinnigen, mit Hilfe materieller Kräfte erzeugt“". Als Julian auf seine Frage, wer denn mit dieser Anspielung gemeint sei, von Eusebios auf den großen, in Ephesos wirkenden Theurgen Maximus verwiesen wurde, soll er sich mit den Worten: „Bleibe du bei deinen Büchern, mir hast du gesagt, was ich hören wollte“ von seinem Lehrer verabschiedet haben, um nach Ephesos aufzubrechen.

$11 \mathrm{Zu}$ Maximus siehe Eunapius, Vitae sophistarum VII, 1-5 (473-481) und Prosopographie I (Anm. 1), sub voce Maximus of Ephesus 21.

12 Libanius, Oratio XVIII, 18.

13 Iulianus, Epistula ad Themistium 253 B; Epistula ad Athenienses 275 B-277 A. 
II., ihn zu seinem Mitregenten und präsumtiven Nachfolger und übertrug ihm die Aufgabe, die kaiserliche Gewalt am Rhein und im Westen des Reiches zu vertreten. Damit nahm er eine Stellung ein, die einem alten Traum der Philosophen die Chance einer Realisierung zu bieten schien: Dass die politischen Verhältnisse sich erst dann zum Besseren wendeten, wenn die Könige Philosophen oder die Philosophen Könige würden. ${ }^{14}$ Julian war kaum in Gallien angelangt, da empfing er von Themistios, einem seiner Lehrer, bei dem er Platons Nomoi studiert hatte, ${ }^{15}$ eine Denkschrift, in der er sich unter Berufung auf Aristoteles aufgefordert sah, von der philosophischen Theorie zur politischen Praxis voranzuschreiten und, wie es im Anschluss an Aristoteles' Politik heißt, zum Architekten edler Werke zu werden. ${ }^{16}$ Julian kannte seinen Aristoteles, und er hielt Themistios vor, dass der Meister mit dem „Architekten edler Werke“ nicht den Praktiker der Politik, den König oder Staatsmann meint, sondern den Gesetzgeber und Ratgeber, die beide auf indirekte Weise für das Wohl des Staates wirken, indem sie der praktischen Politik durch Beratung und Ausarbeitung der gesetzlichen Grundlagen des Staates die Richtung weisen. Doch obwohl Julian, auch unter Berufung auf Aristoteles, am Vorrang der Theorie und Kontemplation prinzipiell festhält, erklärt er sich in seiner Antwort an Themistios bereit, die Rolle, die ihm das Schicksal aufgedrängt habe, anzunehmen, und er bittet Gott und die Philosophen, an deren Stelle er sich auf den Platz eines Herrschers gestellt sieht, um Hilfe und Unterstützung. ${ }^{17}$ Damit ist das Motiv angeschlagen, das sein Selbstverständnis als Kaiser vom Anfang bis zum Ende bestimmt: Sein Kaisertum steht im Dienste seines Gottes und der neuplatonischen Philosophie, die er in Pergamon, Ephesos und Athen in sich aufgesogen hat.

Einem Herrscher aus dem Geist der Philosophie Platons war aufgegeben, bei der politischen Gestaltung der menschlichen Verhältnisse der Idee der Gerechtigkeit zu folgen. Was dies bezogen auf das platonische Staatsmodell und auf die Lebenswirklichkeit des spätantiken Römischen Reiches bedeutete, hat Julian in seinem im Winter 358/59 verfassten zweiten Enkomion auf Kaiser Constantius

14 Plato, Politeia 473 d; Epistula 10, 326 a.

15 Iulianus, Epistula ad Themistium 257 D; zu seiner Person siehe Prosopographie (Anm. 1), S. 889-894, sub voce Themistius 1.

16 Iulianus, Epistula ad Themistium 263 C-D mit Bezug auf Aristoteles, Politika 1325 b. Der communis opinio zufolge gehört das Sendschreiben in die Anfänge seiner Alleinherrschaft: siehe Athanassiadi-Fowden, Polymnia, Julian and Hellenism. An Intellectual Biography, Oxford 1981, S. 90-96 und Criscuolo, Ugo, Sull'epistola di Giuliano al filosofo Temistio, in: Koinonia 7, 1983, S. 90-96. Doch spricht für eine Datierung in das Jahr 356 nicht nur die vorausgesetzte Lebenssituation Julians, der Übergang von einem philosophischen Studien gewidmeten Privatleben zu dem tätigen Dasein des Herrschers, sondern auch die in der Antwort an Themistios mehrfach wiederholte Berufung auf seine völlige Unerfahrenheit in öffentlichen Geschäften. Hinzu kommt, dass er am Schluss seines Antwortschreibens Gott und nicht die Götter beziehungsweise einen bestimmten heidnischen Gott mit Namen anruft. Dies geschah nur, solange er seinen Abfall vom Christentum mit Rücksicht auf Constantius verbergen musste.

17 Iulianus, Epistula ad Themistium 266 D-267 B. 
dargelegt. ${ }^{18}$ Darin entwickelt er anhand der Dichotomie der beiden Stände, Armee und Zivilbevölkerung, die Funktion gerechter Herrschaft nach Analogie der Arbeitsteilung in einer Herde. Der Kriegerstand sorgt wie die Hunde einer Herde für den Schutz vor äußeren Feinden, während die Produzenten für den Unterhalt ihrer Beschützer aufzukommen haben. Aufgabe des Herrschers ist es, dafür zu sorgen, dass jeder Stand die ihm zugeordnete Funktion wahrnimmt, und zu verhindern, dass die Untertanen einander Unrecht tun, indem die Produzenten dem Kriegerstand die Versorgung verweigern oder dieser, statt seine Schutzfunktion wahrzunehmen, über die Produzenten herfällt und sich nimmt, was er will, sodass die staatliche Ordnung darüber zerbricht. ${ }^{19}$

Es wäre nicht schwer zu zeigen, dass ein großer Teil der Regierungstätigkeit Julians in der Zeit seiner Alleinherrschaft, soweit sie in der Rechtssammlung der Kaisergesetze, dem Codex Theodosianus, dokumentiert ist, unter das dargelegte Grundprinzip der gerechten Herrschaft subsumiert werden kann. ${ }^{20}$ Das kann und braucht hier nicht zu geschehen, und es soll genügen, ein Beispiel aus der Zeit seiner Mitregentschaft in Gallien heranzuziehen. Im Jahre 358 n.Chr. nahm Julian einen Konflikt mit dem von Kaiser Constantius eingesetzten Chef der Zivilverwaltung, dem Praetorianerpräfekten Florentius, auf sich, um zu verhindern, dass die ohnehin schwer belastete Zivilbevölkerung einen Aufschlag auf den regulären Steuersatz zahlen musste. ${ }^{21}$ Julian hielt ihn für unnötig und legte eine eigene Berechnung des Bedarfs vor, doch Florentius bestand auf seinen Ansatz und appellierte an Kaiser Constantius. Am Ende setzte sich Julian durch, aber ohne Folgen blieb der Konflikt für ihn nicht. Constantius ordnete eine Untersuchung des Falls an, Julians engster Berater und väterlicher Freund, der Neuplatoniker Saturninius Secundus Salutius, wurde von dem hohen Amt, das er an seinem Hofe bekleidete, abberufen und nach Kleinasien verbannt. ${ }^{22}$ Julian selbst fürchtete damals um sein Leben. In einem Brief, den er an einen anderen Freund und Berater aus dem Kreis

18 Zum Folgenden vgl. Iulianus, Oratio II, 86 D-91 D; zur Datierung der Rede vgl. Bowersock, Glen W., Julian the Apostate, Oxford 1978, S. 43 Anm. 10.

19 Iulianus, Oratio II, 86 D-91 D.

20 Näheres dazu bei Bringmann, Klaus, Kaiser Julian, Darmstadt 2004, S. 60 ff.; 93 ff.; 153 ff.

21 Vgl. Bringmann, Klaus (Anm. 20), S. 61 f.; zu Florentius siehe Prosopographie I (Anm. 1), S. 365, sub voce Flavius Florentius 10.

$22 \mathrm{Zu}$ seiner Person und Karriere siehe Prosopographie I (Anm. 1), S. 814-817, sub voce Saturnius Secundus Salutius 3. Ob er oder Flavius Sallustius, auch er ein Heide und von Julian im Jahre 361 zum Praetorianerpraefekten des Westens ernannt (Prosopographie I, S. 797 f., sub voce Flavius Sallustius 5), der Verfasser des neuplatonischen Traktats Über die Götter und den Kosmos ist, ist strittig. Julian verfasste im Winter 358/59, um seinen väterlichen Freund zu ehren und sich selbst zu trösten, die Trostschrift an sich selbst anlässlich der Abberufung des Salutius: Iulianus, Oratio VIII. Über sein Verhältnis zu dem älteren Freund heißt es in $242 \mathrm{C}$ : „Nicht durch Eide oder derartige Bande beglaubigten wir unsere Freundschaft, wie es Theseus oder Perithous taten, sondern stillschweigend auf der Grundlage gemeinsamer Überzeugungen und Absichten waren wir wie selbstverständlich so weit entfernt davon, irgendeinem Bürger zu schaden, dass wir gar nicht darüber zu beratschlagen brauchten." 
der Neuplatoniker, den Arzt Oreibasios von Pergamon, ${ }^{23}$ richtete, rechtfertigte er seine unnachgiebige Haltung unter Berufung auf Platon und Aristoteles, die beiden Philosophen, deren Schriften seinem Studium der Philosophie bei den Neuplatonikern zugrunde gelegen hatten. Er schreibt: „Durfte er [der Schüler des Platon und des Aristoteles] ruhig zusehen, wie man unglückliche Menschen diesen Dieben [den korrupten Steuereintreibern] auslieferte, oder musste er ihnen nicht nach Kräften beistehen? ${ }^{24}$ Dann gibt er zu erkennen, was ihn dazu brachte, im Konflikt mit dem Praetorianerpräfekten sein Leben zu riskieren. Es war der Glaube an eine Berufung: Gott selbst, so sagt er, hat ihn auf den Platz eines Herrschers gestellt, damit er mit dessen Hilfe dem Unrecht wehre. Seine Worte lauten:

Nun, ich halte es für schmählich, dass man Militätribune, wenn sie, wenn auch mit triftigem Grund, die Kampflinie verlassen, auf der Stelle hinrichtet und sie nicht einmal ein Begräbnis erhalten, selbst aber aus der Kampflinie für diese unglücklichen Leute [die von der Zusatzsteuer bedroht sind] ausschert, obwohl doch der Gott, der uns auf diesen Platz gestellt hat, unser Mitkämpfer ist. Doch wenn es sich fügen sollte, deswegen das Äußerste zu erleiden, so wäre es kein geringer Trost, mit dem Bewusstsein, das Schöne und Gute getan zu haben, die Reise [ins Jenseits] anzutreten. ${ }^{25}$

Unverkennbar stilisiert sich Julian mit diesen Worten als Gefolgsmann des Sokrates, sowohl der Lehre, dass es besser ist Unrecht zu leiden als Unrecht zu tun, als auch der Haltung, die Sokrates im Angesicht des Todes an den Tag gelegt hatte. Er bewährte die Tugend der Gerechtigkeit und der Tapferkeit und, indem er dies tat, auch die der Frömmigkeit; denn er folgte dem Gott, der ihn auf den Platz eines Vorkämpfers für die Gerechtigkeit gestellt hatte. Diese Selbstdarstellung hatte Außenwirkungen. In den eingangs zitierten Inschriften wird Julian als magister omnium virtutum und als ein Herrscher aus dem Geist der Philosophie vorgestellt, der in der Praktizierung der Gerechtigkeit und der übrigen Tugenden ihren inneren Zusammenhang erfasst hat. Dem Gott und den Philosophen, deren Jünger er war, zu folgen waren für ihn zwei Seiten einer Medaille. Als er den Neuplatoniker Priscus einlud, zu ihm an seinen Hof nach Gallien zu kommen, geschieht das in einem feierlichen Schwur, mit dem er sein Leben dem Dienst für das Anliegen der wahren Philosophen weiht. Seine Worte lauten:

Ich schwöre dies beim Urheber alles Guten, das mir zuteil geworden ist, und bei meinem Erretter, dass ich mir nur deshalb das Leben wünsche, um euch in einer gewissen Sache nützlich sein zu können. Wenn ich ,euch'sage, meine ich die wahren Philosophen, und du weißt, wie sehr ich dich zu ihnen zähle. ${ }^{26}$

23 Zu seiner Person siehe Prosopographie I (Anm. 1), S. 653 f., sub voce Oribasius und Schröder, Heinrich Otto, Realencyclopädie der classischen Altertumswissenschaft Supplement VII (1940), S. 797-812. Er begleitete Julian als Leibarzt und Bibliothekar nach Gallien und blieb in seiner Nähe bis zu dessen Tod in Mesopotamien.

24 Iulianus, Epistula 14 Bidez/Cumont, 385 B.

25 Iulianus, Epistula $14 \mathrm{Bidez} /$ Cumont, $385 \mathrm{C}-\mathrm{D}$.

26 Iulianus, Epistula $13 \mathrm{Bidez} /$ Cumont. 
Der Brief wurde geschrieben, nachdem Julian von einer schweren Erkrankung, die ihn im Winter 358/59 n.Chr. befallen hatte, genesen war. Unter dem Eindruck der existenziellen Gefährdung, die ihm der Konflikt mit Florentius eingebracht hatte, ging er damals daran, seine Erhebung zum Augustus vorzubereiten und Constantius offen herauszufordern. Dabei war dem Netzwerk der heidnischen Neuplatoniker eine wichtige Rolle zugedacht. Nach Julians Tod hat einer der Hauptbeteiligten des Komplotts, der bereits erwähnte Oreibasios von Pergamon, eine Darstellung der Erhebung in Gallien gegeben, die der Historiker Eunapios für sein Geschichtswerk benutzt hat. Zwar ist dieses Werk als ganzes verloren, aber Eunapios erwähnt in seinen Lebensbeschreibungen der Sophisten unter Hinweis auf sein Geschichtswerk, dass Julian, der sich während seines kurzen Studienaufenthaltes in Athen im Sommer 355 in die Eleusinischen Mysterien hatte einweihen lassen, vor der arrangierten Ausrufung zum Augustus den Hierophanten der Eleusinischen Mysterien nach Gallien kommen ließ und mit dessen Unterstützung die geheimen Opferriten zelebrierte, die ihm die Hilfe der Gottheit sichern sollten. ${ }^{27}$ Der Hierophant war nicht der einzige, der damals nach Gallien gerufen wurde. Aus Athen kam, vielleicht zusammen mit dem Hierophanten, auch der Neuplatoniker Priscus, den er in Athen kennengelernt hatte. Seitdem gehörte dieser zum engsten Freundes- und Beraterkreis Julians, er begleitete ihn auch auf den persischen Feldzug und war zugegen, als Julian starb. ${ }^{28}$ Alle Vorbereitungen, die Julian im Jahre 359/60 betrieb, waren geheim, und so ist denn auch der an Priscus gerichtete Einladungsbrief ein Dokument der Verhüllung. Anstelle des Gottesnamens verwendet Julian Prädikationen, die auch für den Gott der Christen gebräuchlich waren. Die dezidiert christenfeindlichen Neuplatoniker, die er meint, heißen , wahre Philosophen', und um welcher Sache willen er sein Leben ihnen zu weihen gelobt, bleibt unausgesprochen. Briefe konnten abgefangen werden, und in Anbetracht des Kontroll- und Spitzelsystems, das der von Usurpationen bedrohte Constantius errichtet hatte, waren Vorsicht und Verstellung überlebenswichtig. Julian war damals, wie Libanios sich ausdrückt, ein hellenischer, sprich: heidnischer Löwe in christlicher Eselshaut. ${ }^{29}$ Angesichts des engen Bündnisses, das die von ihm als die wahren Philosophen apostrophierten Neuplatoniker mit der Sache des heidnischen Götterglaubens geschlossen hatten, und der Erfahrung der Bedrohung durch das seit der konstantinischen Wende von Staats wegen geförderte Christentum kann jedoch meines Erachtens kein vernünftiger Zweifel daran bestehen, dass Julian an der zitierten Briefstelle auf das gemeinsame Anliegen einer Neubelebung des Götterglaubens aus dem Geist des Neuplatonismus

27 Eunapius, Vitae sophistarum VII, 3,6-9 (476); zu den Quellenverhältnissen vgl. Chalmers, W., Eunapius, Ammianus, and Zosimus, in: Classical Quarterly N.S. 13 (1960), S. 152 ff. und Bowersock (Anm. 16), S. 7 f. mit Anm. 10.

$28 \mathrm{Zu}$ Priscus siehe Prosopographie I (Anm. 1), S. 730, sub voce Priscus 5. Eunapios weiß zu berichten, dass zusammen mit dem Hierophanten von Eleusis ein gewisser Euhemerus an Julians Hof nach Gallien kam (siehe Anm. 27). Dieser Euhemerus ist nicht in die Prosopographie I aufgenommen.

29 Libanius, Oratio XVIII, 19. 
anspielt. Was Julian als das Hauptübel seiner Zeit beklagte, war die Gottesferne, und diese manifestierte sich für ihn im Abfall von den Göttern der Vorfahren und in der Vernachlässigung ihrer Kulte. Das Christentum, das sich auf Kosten der Altgläubigen ausbreitete, war der Hauptfeind, und was er von diesem dachte, hat er später im Einleitungssatz seiner Schrift Gegen die Galiläer schnörkellos zu Protokoll gegeben: „Richtig erscheint es mir, die Gründe darzulegen, weswegen ich überzeugt bin, dass das Gedankengebäude der Galiläer ein Machwerk von Menschen in betrügerischer Absicht darstellt. “30 Julian bezeichnete die Christen als gottlos beziehungsweise götterlos, weil sie entweder von den Göttern Griechenlands und Roms oder von dem Gott der Juden abgefallen seien und den Leichnam eines Gekreuzigten anbeteten. Paulus galt ihm als Betrüger, und er nahm schweren Anstoß an der Theologie des Johannesevangeliums, die auf dem für einen Neuplatoniker unannehmbaren Axiom beruhte, dass das Vernunftprinzip des Logos in der Person Jesu Fleisch geworden sei. ${ }^{31}$

Der grundsätzlichen Ablehnung des Christentums als einer Afterreligion entsprach die Sensibilisierung für die pragmatischen Gründe, die es zum Gewinner der diagnostizierten Gottes- und Götterferne gemacht hatten. Da waren die Fürsorge für die Armen und die schwächsten Glieder der Gesellschaft, die Witwen und Waisen, die an die städtische Struktur des Römischen Reiches angelehnte Organisation der christlichen Kirchen und die Rolle des Klerus als einer geistlichen Führungselite. Dies alles versetzte das Christentum nach Julians Überzeugung in die Lage, von der verbreiteten Gleichgültigkeit gegenüber den heidnischen Kulten zu profitieren. Abgesehen von der Minderheit, die dem herrschenden Zeitgeist trotzte und an einem praktizierenden Heidentum festhielt, waren es eigentlich nur die Juden, die er von dem Verdikt der Gottesferne ausnahm. Er schätzte an ihnen die Treue zu dem Gott ihrer Vorfahren, und er war, nachdem er die Alleinherrschaft gewonnen hatte, auch bereit, ihnen den Tempel in Jerusalem zu restituieren und das Judentum so in den Kreis der Opferreligionen wieder zurückzuholen. Was er an ihnen zu tadeln hatte, war lediglich das Paradoxon, dass sie ihren Stammesgott mit der höchsten Gottheit schlechthin verwechselten. ${ }^{32}$ Richtige Vorstellungen von der Einheit und Vielfalt der Götter zu besitzen war für Julian die höchste theoretische Leistung der neuplatonischen Philosophie - so, wie es die praktische der ihm von Maximus von Ephesos vermittelten Theurgie war, von den Göttern Zeichen zu empfangen und in Träumen und Visionen mit ihnen in Verbindung zu treten. Er glaubte sich von den Göttern zur Herrschaft über den Erdkreis berufen, um eine Mission zu erfüllen, und diese Mission bestand, um seine eigenen Worte zu gebrauchen, in der Befreiung der

30 Iulianus, Contra Galilaeos 39 A-B.

31 Iulianus, Contra Galilaeos 201 E-202 A und 191 D-E; zu Julians Urteil über den Apostel Paulus vgl. 199 A und 106 B; zur Kritik am ersten Kapitel des Johannesevangeliums siehe 327 B.

$32 \mathrm{Zu}$ Julian und die Juden vgl. die grundlegende, materialreiche Untersuchung von Adler, Michael, The Emperor Julian and the Jews, in: Jewish Quarterly 5 (1893), S. 591-651; deutsch in: Julian Apostata, Hg. Richard Klein (Wege der Forschung 509), Darmstadt 1978, S. 48-111. 
Welt vom Schmutz der Gottlosigkeit. ${ }^{33}$ Als er zu Beginn seiner Alleinherrschaft in seinen Caesares die Regierungsgrundsätze seiner Vorgänger durchmusterte, blieb als Vorbild nur Marc Aurel, der Philosoph auf dem Kaiserthron. Der Grund ist, dass er als einziger sich dem Prinzip verschrieben hatte, das in der Hierarchie der Werte den höchsten Rang innehatte: Marc Aurel folgte den Göttern, und das hieß: er ahmte ihre Bedürfnislosigkeit und ihre Fürsorge für die Menschheit nach. ${ }^{34}$ Retter und Wohltäter zu sein bezog sich auf alle Felder menschlicher Existenz, aber die höchste Aufgabe bestand nach Julians Zeitdiagnose in der Wiederherstellung des Götterglaubens. Indem er seine Alleinherrschaft diesem höchsten Ziel widmete, diente er zugleich der Sache der ,wahren“ Philosophen, als deren Werkzeug er sich betrachtete. ,Und obwohl ich, wie du weißt, an äußeren Gütern der Überlegene bin," schreibt er einmal, „unterwerfe ich mich meinem Lehrer, seinen Freunden und Altersgenossen sowie den Philosophen seiner Schule ...". ${ }^{35}$ Der Lehrer, der hier gemeint ist, ist kein anderer als Maximus von Ephesos. Er gehörte zu denen, die er unmittelbar nach dem Tod des Constantius an seinen Hof rief. Bei dessen Eintreffen in Konstantinopel unterbrach er eine Senatssitzung, um ihn überschwenglich zu begrüßen. ${ }^{36}$ Maximus wurde wie Priscus sein Berater und blieb in seiner engsten Umgebung bis zu seinem Tod im Zweistromland. Beide bildeten zusammen mit dem Kaiser das neuplatonische Dreigestirn, das im Mittelpunkt eines Netzwerks heidnischer Philosophen und Rhetoren stand und diese in die Pflicht zu nehmen suchte, bei der Neubegründung des Götterglaubens aus dem Geist des Neuplatonismus an prominenter Stelle mitzuwirken. ${ }^{37}$

33 Vgl. Julians Gebet an die große Göttermutter (Oratio V, 180 B): ,... gib allen Menschen das Heil, dessen Hauptstück die Erkenntnis der Götter ist, vor allem aber dem römischen Volk insgesamt, dass es sich vom Schmutz der Gottlosigkeit befreie ..." Zu Julians Glauben an seine göttliche Berufung vgl. den Mythos in seiner Antwort an den Kyniker Herakleios: Oratio VII, $233 \mathrm{~A}-234 \mathrm{C}$.

34 Iulianus, Caesares 333 B-335 A.

35 Iulianus, Oratio VII, $235 \mathrm{C}-\mathrm{D}$.

36 Vgl. Libanius, Oratio XVIII, 155 f. und Ammianus Marcellinus, Res gestae XXII, 4,1. Während Libanios den Empfang des Maximus dahingehend deutet, dass Julian damit die Philosophie über die kaiserliche Würde gestellt habe, kritisiert Ammianus Marcellinus das Verhalten Julians als eines Kaisers unwürdig.

37 Als Julian nach Gewinnung der Alleinherrschaft Maximus an seinen Hof rief, zeigte er sich erleichtert darüber, dass sein verehrter Mentor trotz seiner vielfältigen Aktivitäten, mit denen er sich im Herrschaftsbereich des christlichen Kaisers Constantius für die gemeinsame Sache eingesetzt hatte, nichts Ernstliches zugestoßen war: vgl. Iulianus, Epistula 26 Bidez/Cumont, 415 A-B. Als er Maximus schrieb, war der ihm eng verbundene Rhetor Euagrios schon bei ihm eingetroffen: Iulianus, Epistula 26 Bidez/Cumont, 415 D (dieses Zeugnis fehlt in Prosopographie I (Anm. 1), S. 285, sub voce Euagrius 4). Noch vor dem Tod des Constantius bot Julian dem Sophisten Theodoros (siehe Prosopographie I (Anm. 1), S. 897, sub voce Theodorus 8) an, dass sie sich auf der Grundlage gemeinsamer Überzeugungen gegenseitig unterstützten (Iulianus, Epistula $30 \mathrm{Bidez} / \mathrm{Cumont}$ ) und ernannte ihn später zum Hohenpriester der von ihm gegründeten heidnischen Staatskirche (das Wort der Kürze halber) in der Provinz Asia. Einladungen ergingen auch an zwei Philosophen aus der Schule des Jamblichos, an Eustathios (siehe Prosopographie I (Anm. 1), S. 310, sub voce Eustathius 1) und an Chrysanthios (siehe Prosopographie I (Anm. 1), S. 202 f. sub voce Chrysanthius of Sardis) sowie an den gefeierten Rhetor 
Als Julian seinen Oberkämmerer Eutherius aufforderte, den Göttern ein Dankopfer für die unblutige Gewinnung der Alleinherrschaft darzubringen, machte er deutlich, dass dies auch zum Dank für die Rettung der Sache aller Hellenen, das heißt: für die Rettung des Götterglaubens, geschehen sollte. ${ }^{38}$ Und an seinen Onkel Iulianus, den Bruder seiner Mutter, schrieb er zur Rechtfertigung seiner Usurpation: „Warum ich also gekommen bin? Weil die Götter es mir ausdrücklich befohlen haben und mir, wenn ich gehorchte, Heil und Rettung, wenn ich aber [in Gallien] bliebe, ein Schicksal ankündigten, das keiner unter den Göttern wahr machen möge. ‘39

Was aus diesem Ansatz für die Regierungspraxis folgte, kann und braucht hier nicht im einzelnen beschrieben zu werden. ${ }^{40}$ Nur soviel sei gesagt: Julian sorgte für die Erneuerung heidnischer Kulte, für die Rückgabe von Tempeln und Tempelgütern, und er traf Maßnahmen, die der Marginalisierung des Christentums dienten. Den Kirchen und dem Klerus wurden Privilegien entzogen, die innerchristlichen Gegensätze geschürt, Christen vom höheren Lehramt ausgeschlossen, und die höheren Bildungsanstalten, die Schulen der Grammatiker und Rhetoren, deren Absolvierung die Voraussetzung für den Zugang zu den Führungspositionen in der Reichsadministration und in den Städten war, sollten zu heidnischen Gesinnungsschulen gemacht werden. Diesen gegen die Christen gerichteten Maßnahmen entsprach die Förderung des Heidentums. Julian versuchte, aus der Zersplitterung und Vielgestaltigkeit, in der sich die heidnischen Kulte präsentierten, eine vielgestaltige Einheit zu schaffen, und er wandte dabei Methoden an, die dem bekämpften Feind, dem Christentum, entlehnt waren. Julian begann damit, eine die Provinzen des Reiches umspannende heidnische Kirchenorganisation zu gründen, er initiierte und alimentierte eine heidnische Sozialfürsorge, und er war bemüht, das moralische und intellektuelle Niveau der Priesterschaften zu heben und an die Stelle der alten Honoratiorenverwaltung der sacra einen heidnischen Klerikerstand zu setzen, der an der Philosophie der Neuplatoniker geschult die Funktion einer geistigen und geistlichen Elite wahrzunehmen in der Lage war. Es war nur folgerichtig, dass er prominente Neuplatoniker in die Führungspositionen seiner heidnischen Staatskirche zu berufen suchte. Er schrieb Enzykliken und theologisch-philosophische Traktate, und noch auf dem Marsch nach Mesopotamien trug er sich mit dem Gedanken, ein Rundschreiben über wahre Frömmigkeit zu verfassen.

Die Geschichtswissenschaft kann die Frage nicht schlüssig beantworten, ob Julian mit seiner Religionspolitik zum Ziel gelangt wäre, wenn seine Alleinherr-

Himerios (siehe Prosopographie I (Anm. 1), S. 436, sub voce Himerius 2: Iulianus, Epistula 38 Bidez/Cumont). Maximus und Chryanthios wurden zunächst dazu ausersehen, die Wiederaufnahme der heidnischen Kulte in Griechenland zu organisieren. Chrysanthios entzog sich dieser Aufgabe, ließ sich aber später dafür gewinnen, zusammen mit seiner Frau Melite hohepriesterliche Würden in Lydien zu übernehmen: vgl. Eunapius, Vitae sophistarum VII, 4,9 (478).

38 Iulianus, Epistula $29 \mathrm{Bidez} / \mathrm{Cumont}$.

39 Iulianus, Epistula $28 \mathrm{Bidez} / \mathrm{Cumont}$.

40 Vgl. dazu Bringmann (Anm. 20), S. 98 ff.; 120 ff.; 129 ff. 
schaft nicht zwanzig Monate, sondern vierzig Jahre wie die des Augustus gedauert hätte. Es versteht sich von selbst, dass er schnelle und leichte Siege bei Opportunisten und Karrieristen gewann. Derartige Leute schlagen sich immer auf die Seite der herrschenden Macht, und es kann nicht verwundern, dass er sie auch in den Reihen des christlichen Klerus fand. ${ }^{41}$ Aber Julian wollte sich damit nicht zufrieden geben, er wollte gläubige, fromme Verehrer der Götter, und das gegen den passiven Widerstand zu erreichen, der ihm von christlicher Seite und von der Masse der religiös Indifferenten geleistet wurde, erwies sich in der Kürze der Zeit, die ihm blieb, als unmöglich. Schon als er im Sommer 362 durch Kleinasien nach Antiocheia in Syrien reiste, musste er erleben, dass sein religiöser Eifer vielerorts ins Leere stieß und die erhoffte Resonanz der Bevölkerung ausblieb. Einem seiner Gesinnungsfreunde, dem Philosophen Aristoxenos, der im Begriff war, ihm in Kappadokien entgegenzureisen, schrieb er:

Suche mich also in Tyana zu erreichen, bei Zeus, dem Schutzherrn der Freundschaft, und zeige uns unter den Kappadokern (in deiner Person) einen echten Hellenen [das heißt einen gläubigen Heiden]; bisher sehe ich hier nämlich nur Menschen, die nicht opfern wollen, auf der anderen Seite aber nur einige wenige, die zwar wollen, aber sich nicht darauf verstehen. ${ }^{42}$

In Antiocheia waren dann die Erfahrungen, die er mit dem Programm einer Wiederbelebung des Heidentums machte, womöglich noch niederschmetternder, ${ }^{43}$ und der Bürgerschaft der ägyptischen Metropole Alexandreia schickte er eine geharnischte Botschaft, weil sie nicht tun wollte, was in seinen Augen das Selbstverständliche war: Die Alexandriner weigerten sich, König Helios, seinen Schutzgott und universalen Wohltäter der Menschheit, zu verehren. Er schrieb:

Wie solltet ihr jene anderen Segnungen nicht kennen, die Tag für Tag nicht nur einzelnen Menschen, einem einzelnen Volk, einer einzelnen Stadt, sondern ganz allgemein der gesamten Welt von den sichtbaren Göttern geschenkt werden? Seid ihr als einzige bar des Empfindens für den von Helios niederströmenden Glanz? Wisst ihr als einzige nicht, dass durch ihn Sommer und Winter entstehen? Ist euch als einzigen unbekannt, dass alles Leben von ihm geschaffen, alle Bewegung von ihm hervorgerufen wird? Nehmt ihr nicht wahr, wie große Segnungen Selene [die Mondgöttin], die aus Helios und durch ihn Schöpferin aller Dinge [in der sublunaren, irdischen Welt] ist, für eure Stadt bewirkt? Und da wagt ihr es, keiner dieser Gottheiten eure Verehrung zu bezeugen, glaubt aber, dass Jesus, den weder ihr noch eure Väter gesehen haben, der Gott Logos sein müsse? Den aber, der von Anbeginn der Zeiten das ganze

41 Bekannt ist der Fall des Bischofs Pegasios von Ilion, der sich der heidnischen Staatskirche Julians zur Verfügung stellte und von diesem zu ihrem Priester gemacht wurde: vgl. Iulianus, Epistula 79. Vergleichbar ist der Konfessionswechsel, den Julians gleichnamiger Onkel vom Christentum zum Heidentum vornahm und daraufhin von seinem Neffen zum höchsten Verwaltungsbeamten der Diözese Oriens ernannt wurde: siehe Prosopographie I (Anm. 1), S. 470 f., sub voce Iulianus 12.

42 Iulianus, Epistula 78 Bidez/Cumont. Julian scheint beabsichtigt zu haben, dem Philosophen die Erneuerung des Opferkultes in Tyana zu übertragen.

43 Vgl. Bringmann, Klaus (Anm. 20), $161 \mathrm{ff}$. 
Menschengeschlecht sieht und erblickt und verehrt und dabei gedeiht, den großen Helios meine ich, das lebende, beseelte, vernunftbegabte Abbild des intellegiblen Vaters, haltet ihr nicht für eine Gottheit? ${ }^{44}$

Obwohl die Enttäuschung darüber, dass sein Enthusiasmus für den neuplatonischen Götterhimmel weder auf überzeugte Christen noch auf indifferente Heiden ansteckend wirkte, ihm in seinen letzten Lebensmonaten hart zusetzte, blieb das Projekt der religiösen Wende bis zuletzt der Leitstern seines Kaisertums. Daran konnte auch die Vorbereitung des Perserkrieges, die seine Arbeitskraft stark beanspruchte, im Grunde nichts ändern. Auch dieser Feldzug gegen den äußeren Feind, dessen der christliche Kaiser Constantius nicht Herr geworden war, war ja dazu bestimmt, durch einen überwältigenden Sieg den Beweis der Gotterwähltheit Julians zu erbringen. Auf dem Marsch nach Mesopotamien gehörte seine Hauptsorge, wo immer er hinkam, der Erfüllung religiöser Pflichten und der Beförderung des frommen Götterglaubens. Davon legt der letzte Brief, den wir von seiner Hand besitzen, beredtes Zeugnis ab. Der Brief ist an seinen Bewunderer und Glaubensgenossen, den Rhetor Libanios, gerichtet und enthält ein Itinerar des in Syrien zurückgelegten Weges. Darin heißt es:

Hier [im syrischen Beroia] hielt ich mich einen Tag auf, besichtigte die Burg, opferte dem Zeus nach Art eines Kaisers einen weißen Stier und führte auch ein kurzes Gespräch mit dem Stadtrat über die Verehrung der Götter. Doch pflichteten meinen Äußerungen zwar alle bei, aber nur wenige ließen sich durch sie wirklich überzeugen, und das waren genau diejenigen, die schon vor meiner Rede gesunder Denkweise zu sein schienen. ${ }^{45}$

Einen Tagesmarsch weiter machte er eine völlig andere Erfahrung:

Batnai also ist trotz der barbarischen Herkunft des Namens ein hellenischer [das heißt: ein heidnischer] Ort; das zeigte sich schon daran, dass allenthalben Weihrauchwolken das Land durchzogen; auch sahen wir überall Opfertiere bereitstehen. Wenn mich das auch überaus erfreute, so erschien es mir doch übertrieben und dem frommen Glauben an die Götter unangemessen. Abseits von der öffentlichen Straße und in der Stille sollten Schlachtopfer für die Götter und religiöse Zeremonien vor sich gehen, indem man nur auf die Sache selbst sieht und keinen anderen Zweck ins Auge fasst. ${ }^{46}$

Julian plante wohl, hierzu einen besonderen Rundbrief zu verfassen, denn er fügt noch hinzu: „Doch dieses Problem wird vielleicht schon demnächst die entsprechende Beachtung [meinerseits] erfahren." Richtig wohl fühlte er sich erst im Umkreis seiner neuplatonischen Glaubensgenossen. Auf seiner nächsten Station, in Hierapolis, nahm er Quartier bei dem Schwiegersohn des älteren Sopatros, eines Schülers des Iamblichos. ${ }^{47}$ Dieser Sopatros, einer der führenden Neuplatoniker im ersten Drittel des vierten Jahrhunderts, hatte am Hof Konstantins des

44 Iulianus, Epistula 111 Bidez/Cumont, 434 B-435 A.

45 Iulianus, Epistula 98 Bidez/Cumont, 399 D.

46 Iulianus (Anm. 45), 400 C-D.

$47 \mathrm{Zu}$ seiner Person siehe Prosopographie I (Anm. 1), S. 846, sub voce Sopater 2. 
Großen als Freund und Berater des Kaisers Einfluss gewonnen, war dann aber einer Hofintrige zum Opfer gefallen und war hingerichtet worden - nach einer Version wegen Propagierung des Götterglaubens, nach einer anderen, weil er durch magische Praktiken eine Hungersnot in Konstantinopel verursacht habe. Wie dem auch sei: Der Neuplatoniker Sopatros war mit der Gloriole eines heidnischen Märtyrers umgeben, und auch der Schwiegersohn hatte sich allen Pressionen der christlichen Kaiser Constantius und Gallus gegenüber standhaft erwiesen und am Götterglauben festgehalten. Hier fand also Julian eine kongeniale Atmosphäre, und die Schilderung, die er Libanios gab, atmet die Begeisterung, die er bei seinem Besuch bei Sopatros' Schwiegersohn empfunden hatte:

Dort [in Hierapolis] kamen uns die Bürger entgegen, und mich selbst nahm ein Gastgeber bei sich auf, den ich zwar zum erstenmal erblickte, der aber schon seit langem von mir geliebt wird. Den Grund dafür kannst du, wie ich wohl weiß, selbst erschließen, doch macht es mir Vergnügen, dennoch davon zu sprechen. Denn immer wieder davon zu hören und darüber zu reden, ist Nektar für mich. Des göttlichen Iamblichos Zögling Sopatros war zugleich der Schwiegervater meines Gastgebers. Denn nicht alles zu lieben, was mit jenen Männern zusammenhängt, scheint mir so schlimm wie kein zweites Unrecht zu sein. Ein Grund, der noch gewichtiger ist, kommt hinzu: Er hat mehrmals meinen Vetter [Kaiser Constantius] und meinen Stiefbruder [den Mitregenten Gallus] bei sich aufgenommen und sich, obwohl er, wie zu erwarten war, vielfach gedrängt wurde, von seiner Verehrung für die Götter abzulassen, von ihrem Wahn nicht anstecken lassen, was schwierig ist. ${ }^{48}$

Der Brief an Libanios gibt einen lebendigen Eindruck von den zwiespältigen Erfahrungen und Gefühlen, denen Julian auf seiner Reise ausgesetzt war. Es gab Städte im Osten des Reiches, in denen die Christen, wenn nicht die Mehrheit, so doch eine starke Minderheit bildeten, unter den Heiden gab es viele, die religiös indifferent waren, und selbst dort, wo wie in Batnai zu Julians Empfang umfangreiche Vorbereitungen getroffen waren, den Göttern zu opfern, nahm er Anstoß an dem ostentativen Übereifer, der ihm nicht recht zu der wahren Frömmigkeit gegenüber den Göttern zu passen schien. Die Frömmigkeit, die er verbreiten wollte, fand er bei den Neuplatonikern, und so kann es nicht verwundern, dass sein Programm der Erneuerung des Götterglaubens in den Inschriften dort die stärkste Resonanz fand, wo er Helfer fand, die ihrerseits vom Geist des Neuplatonismus inspiriert waren. Das gilt insbesondere, aber keineswegs ausschließlich, ${ }^{49}$ für den

48 Iulianus (Anm. 45), 401 B-C.

49 In Thessalonike weihte der Prokonsul von Macedonia Kalliopios einen Altar zu Ehren Julians, „des gottgeliebtesten Erneuerers der Kulte und Heiligtümer“: Conti (Anm. 2), Nr. 54. Kalliopios war Lehrer der Rhetorik und sowohl in Konstantinopel als auch in Antiocheia Kollege des Libanios gewesen: siehe Prosopographie I (Anm.1), 174, Calliopius 2. Er war Heide und stand aller Wahrscheinlichkeit nach ähnlich wie Libanios dem Neuplatonismus nahe. In der Provinz Pannonia secunda, im Gebiet von Mursa (heute Osijek in Kroatien) ist Julian auf einem Meilenstein ,wegen Tilgung der Verfehlungen der Vergangenheit“" geehrt worden: Conti (Anm. 2), Nr. 73. Mit den Verfehlungen der Vergangenheit war, ganz im Sinne Julians, die das Christentum begünstigende Politik seiner Vorgänger gemeint. Veranlasst wurde die Ehrung wohl durch Sex- 
griechischen Osten des Reiches. Beispielsweise haben sich in der Provinz Arabia an der großen Straße von Gerasa nach Philadelphia/Amman und von dort nach Aeropolis mehrere Meilensteine mit Inschriften gefunden, die Akklamationen auf Julian enthalten und sein Kaisertum, auf den ersten Blick überraschend, in Verbindung mit der Losung von einem Gott bringen. Die einschlägigen Akklamatio-

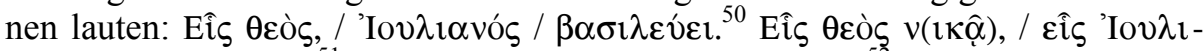

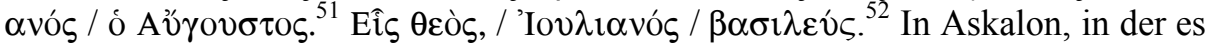
nach Julians Edikt zur Wiederherstellung der heidnischen Tempel und Kulte zu antichristlichen Ausschreitungen gekommen war, ${ }^{53}$ fand sich an der von Gaza nach Berytos führenden Küstenstraße eine Marmorsäule mit der Inschrift: Eîऽ

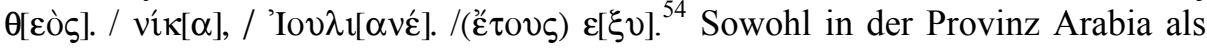
auch in der Provinz Palaestina prima, zu der Askalon gehörte, waren damals engagierte Heiden aus dem Kreis neuplatonischer Intellektueller von Julian zu Statthaltern gemacht worden, in Arabia Belaios ${ }^{55}$ und in Palaestina Leontios. ${ }^{56}$ Libanios nennt beide Sophisten, das heißt, sie waren ähnlich wie er selbst Rhetoren und

tus Aurelius Victor, den Verfasser einer kurzgefassten Römischen Geschichte, den Julian im Jahre 361 zum Statthalter von Pannonia secunda gemacht hatte: Ammianus Marcellinus, Res gestae XXI,10,6. In Africa proconsularis ist im Gebiet von Aradi in Tunesien eine Tafel mit einer Weihung an den „Erhabenen Sonnengott“ zum Wohl des Kaisers gefunden worden: Conti (Anm. 2), Nr. 132, in der Byzacena wurde ein Meilenstein zu Ehren Julians mit einer Strahlenkrone, dem Symbol des Sonnengottes, geschmückt (gefunden im Gebiet von Bordj-BouArreridj/Algerien): Conti (Anm. 2), Nr. 164. Auf einer Statuenbasis in Casae (heute El Mahder/Algerien), in der Provinz Mauretania Stifensis wird Julian als „herausragend in jeder Art der Tugenden“ und „als Erneuerer der Freiheit und der römischen Religion“ gefeiert: Conti (Anm. 2), Nr. 167, und auf der Basis einer Statue, die der Rat von Thibilis (heute Anouna/Algerien) in der Provinz Numidia dem Kaiser gewidmet hat, wird Julian als „Erneuerer der Kulte und Heiligtümer" apostrophiert: Conti (Anm. 2), Nr. 176.

50 Conti (Anm. 2), Nr. 3; alle Meilensteine, die zwischen Gerasa und Aeropolis gefunden worden sind, verzeichnet Dietz, Karlheinz, Kaiser Julian in Phönizien, in: Chiron 30 (2000), S. 836 f. mit dem Text der Akklamationen.

51 Conti (Anm. 2), Nr. 5.

52 Conti (Anm. 2), Nr. 7.

53 Vgl. Stemberger, Günter, Juden und Christen im Heiligen Land, München 1987, S. 158.

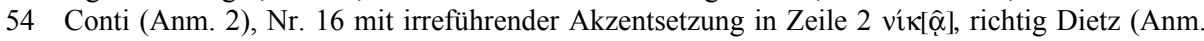
50), S. 837. Die Jahreszahl 465 folgt der mit dem Jahr 104 v.Chr. beginnenden Freiheitsära von Askalon: Sie entspricht dem Zeitraum von Oktober/November 361 bis Oktober/November 362.

55 Prosopographie I (Anm. 1), S. 160, sub voce Belaeus. Er ermutigte die Heiden, sich an den Christen für das unter den christlichen Kaisern erlittene Unrecht zu rächen: vgl. Sartre, Maurice, Bostra, Des origines à l'Islame, Paris 1985, S. 105 f.

56 Prosopographie I (Anm. 1), S. 500, sub voce Leontius 9; strittig ist, ob Leontios in Palaestina prima oder in der südlich angrenzenden Provinz Palaestina Salutaris Statthalter war. Für die erste Möglichkeit spricht der ihm beigelegte Titel consularis, für die zweite wird angeführt, dass er von Libanios gebeten wurde, einem Einwohner von Elusa, einer in Palaestina Salutaris gelegenen Gemeinde, zu helfen: Libanius, Epistula 829. Größeres Gewicht besitzt meines Erachtens der offizielle Titel consularis in einem Erlass Kaiser Julians (Codex Theodosianus XII 1,55). Es empfiehlt sich also, in ihm den Nachfolger des Kyrillos zu sehen, den Julian im Jahre 362 absetzte, weil er Gaza wegen der Ausschreitungen der heidnischen Bevölkerung gegen die Christen bestraft hatte: Prosopographie I (Anm. 1), 237 f., sub voce Cyrillus 1. 
als praktizierende Heiden dem Neuplatonismus verpflichtet. Mit hoher Wahrscheinlichkeit haben sie in ihren Provinzen die Akklamationen der heidnischen Bevölkerung organisiert und die Publikation auf Meilensteinen und sonstigen Schriftträgern veranlasst. ${ }^{57}$ Was aber bedeutet die Propagierung eines Gottes? Sicher ist zunächst, dass sie den vergleichbaren Akklamationen antwortet, mit denen die Christen sich zu ihrem Glauben bekannten. Gerade aus dem syrischen Raum gibt es eine große Zahl epigraphischer Zeugnisse mit Akklamationen von christlicher Seite, in denen die Ein-Gott-Formel auch binitarisch (ein Gott und Christus) oder trinitarisch (ein Gott und Christus und der Heilige Geist) erweitert ist. ${ }^{58}$ Aber wenn das so ist, ergibt sich ein Dilemma, das von Seiten der Althistoriker bisher nicht aufgelöst ist. Einerseits wird die Ein-Gott-Formel mit Julians antichristlichem Kurs verbunden, ${ }^{59}$ andererseits wird ihr ein überkonfessioneller Charakter beigelegt und angenommen, dass durch sie der spätantike Monotheismus als gemeinsames Merkmal von Heidentum und Christentum betont werde. ${ }^{60}$ Dass die Christen den einen Gott verehrten, wurde jedoch von neuplatonischer Seite nachdrücklich bestritten. Julian und die Neuplatoniker warfen den Christen vor, dass sie nicht Gott, sondern tote Menschen, den gekreuzigten Jesus von Nazareth und die Märtyrer, verehrten. Auf heidnischer Seite begegnet die Ein-GottFormel, wie Erik Peterson gezeigt hat, in Verbindung mit einem solaren Henotheismus (der nicht mit einem Monotheismus der strengen Observanz verwechselt werden sollte) beziehungsweise mit dem hellenistisch-orientalischen Aionkult. $^{61}$ Daran konnten die Neuplatoniker anknüpfen, weil sich ihnen in der Vielheit der Götterwelt die Einheit Gottes in der Gestalt des Sonnengottes repräsentierte. In seinem Hymnus auf König Helios, seinen Schutzgott, hat Julian diesem Konzept prägnanten Ausdruck verliehen, indem er von diesem Gott sagt, dass er „,den Himmel mit so vielen Göttern erfüllt, wie er denkend in sich fasst, diese aber sich ungeteilt in ihrer Fülle um ihn scharen und mit ihm in einer Ges-

57 Zur Bedeutung der Akklamationen als organisierter Kundgebungen der Bevölkerung vgl. Roueché, Charlotte, Acclamations in the Later Roman Empire: New Evidence from Aphrodisias, in: Journal of Roman Studies 74 (1984), S. 181-199.

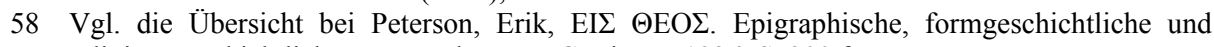
religionsgeschichtliche Untersuchungen, Göttingen 1926, S. 300 f.

59 Vgl. Welles, Bradford, The Inscriptions, in: Gerasa. City of the Decapolis, Hg. C. H. Kraeling, New Haven 1938, S. 355-494, hier S. 489 f.; Balty, Janine und Jean Ch., Julien et Apamée. Aspects de la restauration de l'hellénisme et de la politique anti-chrétienne de l'empereur, in: Dialogue d'histoire ancienne 1 (1974), S. 272-275; Negev, Avraham, The Inscriptions of Wadi Haggag, Sinai, Jerusalem 1977, S. 62-64; Sartre (Anm. 55), S. 105 f.

60 Vgl. Dietz (Anm. 50), S. 837; unklar Roueché (Anm. 57), S. 185: „Bradford Welles, in publishing four of these texts [mit Akklamationen aus der Provinz Arabia], commented that the acclamations 'are a reflection of the emperor's [Julian] fight against Christianity, which used similar slogans', but I would prefer to follow Peterson in seeing these texts simply as a further example of the use of acclamations in such a context."

61 Peterson (Anm. 58), S. 227 ff. Ein gleicher Zusammenhang ist nach Peterson auch für die Verwendung der Formel von seiten eines synkretistischen Judentums anzunehmen: Peterson (Anm. 58), S. $276 \mathrm{ff}$. 
talt verbunden sind““ ${ }^{62}$ Dieses theologische Konstrukt liegt, so scheint mir, den heidnischen Akklamationen mit der Benutzung der Ein-Gott-Formel zugrunde. Sie richtete sich gegen die der Gottlosigkeit beschuldigten Christen, und sie brachte die Gewissheit des Glaubens zum Ausdruck, dass Julian, der Vorkämpfer des einen, die vielgestaltige Götterwelt in sich fassenden Königs Helios den Sieg sowohl über die Christen als auch über die Perser erringen werde. Dass diese Glaubensgewissheit trog, wurde schnell offenbar. Julian kehrte nicht lebend aus Mesopotamien zurück, und den Siegeszug des Christentums hat er nur für kurze Zeit unterbrechen, aber nicht aufhalten können.

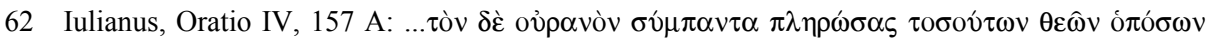

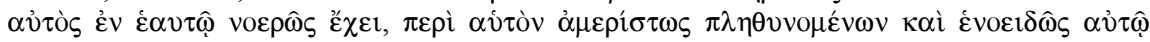
$\sigma v v \eta \mu \mu \varepsilon \dot{\varepsilon} \omega v . .$. 